\title{
Perfis eletrocardiográfico e ecodopplercardiográfico de ovinos após ingestão da suspensão aquosa de Mascagnia rigida Griseb. (Malpighiaceae).
}

\author{
[Electrocardiographical and echocardiographical profiles in experimental \\ Mascagnia rigida Griseb. (Malpighiaceae) toxicosis in sheep] \\ E.P. Lago $^{1}$, M.M. Melo ${ }^{2}$, R.B. Araújo $^{2}$, E.F. Nascimento ${ }^{2}$, E.F. Silva ${ }^{2}$, M.B. Melo ${ }^{3}$ \\ ${ }^{1}$ Departamento de Veterinária - UFV \\ Av. P. H. Rolfs s/n \\ 36570-000 - Viçosa, MG \\ ${ }^{2}$ Escola de Veterinária - UFMG - Belo Horizonte, MG \\ ${ }^{3}$ Aluno de pós-graduação - ICB-UFMG - Belo Horizonte, MG
}

\begin{abstract}
RESUMO
Estudou-se o perfil eletrocardiográfico e ecodopplercardiográfico de ovinos intoxicados experimentalmente pela Mascagnia rigida Griseb (Malpighiaceae). Quinze ovinos machos da raça Santa Inês, com sete meses de idade e pesando, em média, $27 \mathrm{~kg}$, foram distribuídos em três grupos $(\mathrm{G})$ com cinco animais cada: No G1 (controle), os animais receberam apenas água; nos G2, receberam $20 \mathrm{~g} / \mathrm{kg}$ de $M$. rigida durante três dias; e no G3, $20 \mathrm{~g} / \mathrm{kg}$ de $M$. rigida, durante sete dias. As folhas de $M$. rigida foram trituradas com água e administradas na forma de suspensão, por via oral, por meio de sonda. $M$. rigida promoveu aumento da $\mathrm{FC}$ em repouso e, principalmente, durante o esforço físico e diminuiu a fração de ejeção e o percentual de encurtamento sistólico do ventrículo esquerdo.
\end{abstract}

Palavras-chave: ovino, Mascagnia rigida, eletrocardiograma, ecodopplercardiograma

\section{ABSTRACT}

The electrocardiographical and echocardiographical alterations produced by intraruminal administration of Mascagnia rigida griseb. (Malpighiaceae) in sheep were studied. Fifteen male Santa Inês sheep were randomly allotted to three groups: G1 - animals only received water (control); G2 animals were treated with M. rigida, $20 \mathrm{~g} / \mathrm{kg} /$ day, for three days; and $\mathrm{G} 3$ - animals were treated with $\mathrm{M}$. rigida, $20 \mathrm{~g} / \mathrm{kg} /$ day, for seven days. All treatments were administered by an orogastric tube. M. rigida administration produced increase in heart rate during rest and exercise, and decreased in fractional ejection, and fractional shortening.

Keywords: sheep, Mascagnia rigida, electrocardiogram, echocardiogram

\section{INTRODUÇÃO}

No Brasil, são conhecidas 12 espécies de plantas causadoras de morte súbita, das quais se suspeita haver envolvimento cardíaco (Gava et al., 1998; Tokarnia et al., 2000). Dentre elas destaca-se Mascagnia rigida (Malpighiaceae), por ser a espécie mais conhecida do gênero Mascagnia em Minas Gerais, principalmente no norte e nordeste do Estado, onde é conhecida popularmente como salsa-rosa e suma-roxa. Atenção especial tem sido dada a esta planta, pela forma dramática como os casos se apresentam, em que animais aparentemente sadios, quando forçados a movimentar, caem e morrem em poucos minutos. O quadro clínico-patológico sugere que essa planta interfira no funcionamento do coração, causando insuficiência aguda (Melo et al., 2008). Entretanto, até o momento, não foram realizados estudos ecocardiográficos para verificar os efeitos dessa planta na função cardíaca.

Objetivou-se, com esta pesquisa, estudar o perfil cardiográfico de ovinos submetidos à intoxicação experimental por Mascagnia rigida.

Recebido em 9 de julho de 2008

Aceito em 30 de abril de 2009

E-mail: ernanilago@ufv.br 


\section{MATERIAL E MÉTODOS}

Foram utilizados 15 ovinos $^{1}$, machos, da raça Santa Inês, com sete meses de idade e pesando em média $27 \mathrm{~kg}$. Os animais foram alojados em baias de alvenaria e alimentados com capimelefante (Pennisetun purpureum), feno de gramíneas, ração granulada comercial, sal mineral e água.

M. rigida foi utilizada em estado fresco, colhida diariamente no canteiro de plantas tóxicas da Escola de Veterinária - UFMG. Amostras da planta foram enviadas ao Departamento de Botânica - UFMG e identificadas como $M$. rigida Griseb (Malpighiaceae). Uma exsicata foi depositada no Herbário de Botânica - ICBUFMG sob o número BHCB 100819.

Após adaptação por um período de 15 dias, os ovinos foram distribuídos em três grupos de cinco animais: os do G1 (controle) receberam apenas água; os do $\mathrm{G} 2$ receberam $20 \mathrm{~g} / \mathrm{kg}$ de $M$. rigida, durante três dias consecutivos; e os do $\mathrm{G} 3,20 \mathrm{~g} / \mathrm{kg}$ de $M$. rigida, durante sete dias consecutivos. A planta foi administrada via oral, por meio de sonda, após ter sido triturada com água, constituindo, assim, uma suspensão aquosa.

Os animais foram avaliados clinicamente antes da administração da primeira dose da suspensão, o que correspondeu ao tempo zero (T0), e depois, a cada 24 horas, até o oitavo dia, correspondendo, assim, aos tempos T1, T2, T3, T4, T5, T6 e T7. A frequência cardíaca (FC) obtida no exame clínico, em repouso, foi utilizada para comparar com as frequências cardíacas obtidas nos exames eletrocardiográficos (Diniz et al., 2008).

Os exames eletrocardiográficos ${ }^{2}$ foram realizados no tempo zero (T0), após 24 horas (T1) e a cada 48 horas (T3, T5 e T7). Os exames foram realizados com os animais em repouso e depois de serem movimentados (tocados), individualmente, por 10 minutos. Os registros foram feitos segundo Tilley (1992), e o intervalo QT foi corrigido conforme Fridericia (1920).

${ }^{1}$ Aprovado no Comitê de Ética em Experimentação Animal da UFMG - n $032 / 05$.

${ }^{2}$ Eletrocardiógrafo ECG.40 Ecafix-Funbec - São Paulo, Brasil.
Os exames ecodopplercardiográficos ${ }^{3}$ foram realizados no tempo zero (T0), após 72 horas (T3) e 168 horas (T7), e foram gravados em videoteipe $^{4}$. Os posicionamentos e as mensurações seguiram as recomendações de Boon (1998) e Carvalho et al. (2006 e 2007). O experimento foi realizado em delineamento inteiramente ao acaso, em arranjo em parcelas subdivididas, sendo as parcelas os grupos, e as subparcelas os tempos. As variáveis foram comparadas pelo teste Duncan, com 5\% de significância.

\section{RESULTADOS E DISCUSSÃO}

Clinicamente todos os ovinos dos grupos G2 e G3 apresentaram apatia, intolerância ao exercício físico, taquicardia em repouso, inapetência, polaciúria e hipotonia ruminal, sendo que um ovino do G2 morreu após cinco dias. Os cinco animais do G1 não apresentaram alterações clínicas dignas de nota. Essas observações permitem concluir que $20 \mathrm{~g} / \mathrm{kg}$ de folhas de $M$. rigida, trituradas com água e administradas via oral intoxicaram os ovinos deste experimento.

Pela Tab. 1, observa-se aumento significativo da FC, obtida por auscultação durante o exame clínico, com o animal em repouso, nos grupos G2 e G3. Não foram encontrados na literatura estudos sobre a influência de $M$. rigida na FC. Os relatos indicam apenas que, durante a manifestação, da síndrome de morte súbita, a taquicardia ocorria, porém não era possível quantificá-la (Paraguassú, 1983; Tokarnia et al., 1994; Pinheiro et al., 1996).

Os traçados eletrocardiográficos demonstraram que o ritmo cardíaco foi sinusal em todos os animais e não apresentaram alterações que caracterizassem arritmia. A morfologia do complexo P-QRS-T na derivação DII não apresentou alterações que indicassem patologias no tecido de condução.

Em relação ao complexo QRS, houve variação individual em todos os animais, mesmo antes da administração de $M$. rigida. As ondas QS e QR foram as mais frequentes, mas também ocorreram ondas RS e QRS. Outros estudos demonstraram haver variação na morfologia do complexo QRS de ovelhas sadias (Schultz et al.,

${ }^{3}$ HP Sonos 100 CF - Hewlett Packard - Ramsey, EUA.

${ }^{4}$ Sony Brasil Ltda. - Manaus, Brasil. 
1972; Tório et al., 1997). Em ovelhas intoxicadas por plantas contendo glicosídeos cardíacos, anormalidades significativas não foram confirmadas (Botha et al., 1997).

Tabela 1. Frequência cardíaca, obtida por auscultação durante o exame clínico, com animais em repouso, de ovinos do grupo-controle $(\mathrm{G} 1)$ e de ovinos que receberam suspensão aquosa de $M$. rigida Griseb consecutivamente durante três dias (G2) e sete dias G3)

\begin{tabular}{ccccccccc}
\hline \multirow{2}{*}{ Grupo } & \multicolumn{7}{c}{ Tempo após a administração de . rigida } \\
\cline { 2 - 9 } & T0 & T1 & T2 & T3 & T4 & T5 & T6 & T7 \\
\hline G1 & $76,2 \mathrm{aA}$ & $75,6 \mathrm{aB}$ & $74,4 \mathrm{aB}$ & $78,4 \mathrm{aB}$ & $75,2 \mathrm{Ab}$ & $78,0 \mathrm{aB}$ & $78,8 \mathrm{aB}$ & $78,4 \mathrm{aB}$ \\
$\mathrm{G} 2$ & $76,8 \mathrm{eA}$ & $97,2 \mathrm{abA}$ & $99,6 \mathrm{aA}$ & $99,2 \mathrm{aA}$ & $91,0 \mathrm{bcA}$ & $86,5 \mathrm{cdB}$ & $85,0 \mathrm{cdeAB}$ & $80,5 \mathrm{deB}$ \\
$\mathrm{G} 3$ & $76,0 \mathrm{eA}$ & $100,4 \mathrm{abA}$ & $102,8 \mathrm{aA}$ & $97,2 \mathrm{abcA}$ & $96,8 \mathrm{abcA}$ & $96,0 \mathrm{bcA}$ & $93,2 \mathrm{cdA}$ & $88,4 \mathrm{dA}$ \\
\hline
\end{tabular}

Médias seguidas por letras distintas, maiúsculas na coluna e minúsculas na linha, diferem entre si pelo teste Duncan, $(\mathrm{P}<0,05)$. CV: $7,2 \%$.

T0: dia zero; T1: um dia; T2: dois dias; T3: três dias; T4: quatro dias; T5: cinco dias; T6: seis dias; T7: sete dias.

A onda $\mathrm{T}$ apresentou-se bifásica ou positiva, sendo essa variação presente em todos os ovinos de todos os grupos. Estes resultados assemelham-se aos obtidos por outros autores, em que a variação ocorrida na onda $\mathrm{T}$ parece ser uma característica normal do eletrocardiograma de ovinos, que admite essas duas morfologias sem necessariamente indicar doença (Schultz et al., 1972; Tório et al., 1997; Ker et al., 2003). A forma positiva foi predominante após o exercício físico em todos os animais.

Não houve diferença entre os grupos no que se refere às frequências cardíacas, registradas nos eletrocardiogramas, sob condições de repouso (Tab. 2). Apesar disso, é preciso ressaltar que os ovinos de G2 e G3 alcançaram médias muito altas, de 156 e 134 batimentos por minuto (BPM) no T3, quando comparadas com a de 113 BPM ocorrida em G1, o que sugere que $M$. rigida produziu algum efeito sobre a FC. As médias obtidas por auscultação (Tab. 1) reforçam esta sugestão, pois ao se observar que, em G2 e G3, elas foram significativamente maiores que em G1 nos tempos T1, T2, T3 e T4, sendo que, em G3, elas continuaram sendo maiores também nos tempos T5, T6 e T7. Na Tab. 1, observa-se ainda que as médias no $\mathrm{T} 0$ em todos os grupos estavam similares e normais para a espécie, considerada de 70 a 90BPM (Pugh, 2002), e em G1 permanecercem assim até o fim do experimento, enquanto em G2 e G3, após a administração da planta, alcançaram frequências significativamente maiores que o próprio $\mathrm{T} 0 \mathrm{em}$ T1, T2, T3 T4, T5 e T6 (G2) e T1, T2, T3, T4, T5, T6 e T7 (G3), e acima da normalidade em T1, T2, T3 e T4 (G2) e T1, T2, T3, T4, T5 е T6 (G3).

Tabela 2. Frequência cardíaca de ovinos do grupo-controle (G1) e de ovinos que receberam suspensão aquosa de $M$. rigida Griseb consecutivamente durante três dias (G2) e sete dias (G3), registradas no eletrocardiograma durante repouso

\begin{tabular}{cccccc}
\hline \multirow{2}{*}{ Grupo } & \multicolumn{5}{c}{ Tempo após a administração de $M$. rigida } \\
\cline { 2 - 6 } & T0 & T1 & T3 & T5 & T7 \\
\hline G1 & $92 \pm 15,6 \mathrm{aA}$ & $95 \pm 22,9 \mathrm{aA}$ & $113 \pm 12,0 \mathrm{aA}$ & $93 \pm 28,0 \mathrm{aA}$ & $108 \pm 17,4 \mathrm{aA}$ \\
G2 & $104 \pm 5,5 \mathrm{bA}$ & $118 \pm 25,8 \mathrm{bA}$ & $156 \pm 42,2 \mathrm{aA}$ & $102 \pm 16,4 \mathrm{bA}$ & $95 \pm 23,8 \mathrm{bA}$ \\
G3 & $103 \mathrm{~A} \pm 29,4 \mathrm{a}$ & $131 \pm 26,6 \mathrm{aA}$ & $134 \pm 43,0 \mathrm{aA}$ & $124 \pm 21,5 \mathrm{aA}$ & $96 \pm 11,1 \mathrm{aA}$ \\
\hline
\end{tabular}

Médias seguidas por letras distintas, maiúsculas na coluna e minúsculas na linha, diferem entre si pelo teste Duncan, $(\mathrm{P}<0,05)$. CV: 20,0\%.

T0: dia zero; T1: um dia; T3: três dias; T5: cinco dias; T7: sete dias.

Uma explicação para não ter havido diferença estatística entre os grupos, no que se refere às frequências cardíacas em repouso, obtidas pelos eletrocardiogramas, pode ser o estresse ocorrido durante o exame, que pode ter elevado a média da FC do G1, tornando-a similar às médias dos 
outros grupos. Taquicardias durante exames eletrocardiográficos em ovinos normais, em repouso, também foram relatadas (Schultz et al., 1972; Tório et al., 1997; Mir et al., 2000).

Em condições de exercício (Tab. 3), as frequências cardíacas médias dos ovinos do G2 e do G3, obtidas nos eletrocardiogramas, foram estatisticamente maiores que as do G1, em T1 até T5, demonstrando que, nessas condições, $M$. rigida também produz efeito sobre a FC. Na intoxicação por fluoracetato em ovelhas, também foi observado aumento na FC (Schultz et al., 1982).

Tabela 3. Frequência cardíaca de ovinos do grupo-controle (G1) e de ovinos que receberam suspensão aquosa de $M$. rigida Griseb consecutivamente durante três dias (G2) e sete dias (G3), registradas no eletrocardiograma após 10 minutos de exercício

\begin{tabular}{cccccc}
\hline \multirow{2}{*}{ Grupo } & \multicolumn{5}{c}{ Tempo após a administração de $M$. rigida } \\
\cline { 2 - 6 } & T0 & T1 & T3 & T5 & T7 \\
\hline G1 & $125 \pm 18,1 \mathrm{bA}$ & $132 \pm 20,0 \mathrm{abB}$ & $136 \pm 19,7 \mathrm{abB}$ & $119 \pm 16,0 \mathrm{bB}$ & $149 \pm 6,5 \mathrm{aA}$ \\
G2 & $128 \pm 10,9 \mathrm{bcA}$ & $166 \pm 15,2 \mathrm{abA}$ & $184 \pm 33,6 \mathrm{aA}$ & $154 \pm 50,2 \mathrm{bA}$ & $112 \pm 20,6 \mathrm{cB}$ \\
G3 & $121 \pm 29,8 \mathrm{cA}$ & $159 \pm 22,4 \mathrm{abA}$ & $183 \pm 23,5 \mathrm{aA}$ & $152 \pm 23,0 \mathrm{bA}$ & $130 \pm 41,5 \mathrm{cbB}$
\end{tabular}

Médias seguidas por letras distintas, maiúsculas na coluna e minúsculas na linha, diferem entre si pelo teste Duncan, $(\mathrm{P}<0,05)$. CV: $13,7 \%$

T0: dia zero; T1: um dia; T3: três dias; T5: cinco dias; T7: sete dias.

Com relação ao intervalo QT corrigido (QTc), a principal alteração esperada nas doenças cardíacas é que ele esteja aumentado (Tilley, 1992; Koyama et al., 2004). No presente experimento, em nenhum momento, as médias dos intervalos QTe dos grupos tratados foram maiores que as do grupo-controle, tanto em repouso (Tab. 4), quanto em exercício (Tab. 5). Estes resultados indicam que $M$. rigida não causou comprometimento cardíaco suficiente para aumentar o QTc. Entretanto, é preciso ressaltar que a intoxicação em grau discreto pode não ser suficiente para que isso ocorra. Situação semelhante foi demonstrada no experimento de Koyama et al. (2004). Isso sugere que o aumento da FC mascara o aumento do QTe quando a doença está em grau leve e que, só a partir de uma gravidade maior, essa influência é superada, permitindo o prolongamento do QT.

Em outras pesquisas, a duração do intervalo QT em ovelhas normais também variou em função da frequência cardíaca observada (Tório et al., 1997; Mir et al., 2000). Desse modo, pode-se pressupor que $M$. rigida não causou anormalidades no potencial de ação ventricular e, se essas ocorreram, foram muito discretas e não puderam ser demonstradas, provavelmente devido ao aumento na FC.

Tabela 4. Intervalo Q-T corrigido (segundos) de ovinos do grupo-controle (G1) e de ovinos que receberam suspensão aquosa de $M$. rigida Griseb consecutivamente durante três dias (G2) e sete dias (G3), em repouso

\begin{tabular}{cccccc}
\hline \multirow{2}{*}{ Grupo } & \multicolumn{5}{c}{ Tempo após a administração de $M$. rigida } \\
\cline { 2 - 6 } & T0 & T1 & T3 & T5 & T7 \\
\hline G1 & $0,363 \pm 0,02 \mathrm{aA}$ & $0,343 \pm 0,03 \mathrm{abA}$ & $0,309 \pm 0,02 \mathrm{cA}$ & $0,322 \pm 0,02 \mathrm{bcA}$ & $0,334 \pm 0,02 \mathrm{abcAB}$ \\
G2 & $0,326 \pm 0,03 \mathrm{aB}$ & $0,311 \pm 0,01 \mathrm{aB}$ & $0,317 \pm 0,02 \mathrm{aA}$ & $0,333 \pm 0,02 \mathrm{aA}$ & $0,364 \pm 0,03 \mathrm{aA}$ \\
G3 & $0,318 \pm 0,01 \mathrm{aB}$ & $0,284 \pm 0,01 \mathrm{bcB}$ & $0,276 \pm 0,02 \mathrm{cA}$ & $0,304 \pm 0,02 \mathrm{abA}$ & $0,312 \pm 0,02 \mathrm{aB}$
\end{tabular}

Médias seguidas por letras distintas, maiúsculas na coluna e minúsculas na linha, diferem entre si pelo teste Duncan, $(\mathrm{P}<0,05)$. CV: $4,7 \%$.

T0: dia zero; T1: um dia; T3: três dias; T5: cinco dias; T7: sete dias. 
Tabela 5. Intervalo Q-T corrigido (segundos) de ovinos do grupo-controle (G1) e de ovinos que receberam suspensão aquosa de $M$. rigida Griseb consecutivamente durante três dias (G2) e sete dias (G3), após 10 minutos de exercício

\begin{tabular}{cccccc}
\multirow{2}{*}{ Grupo } & \multicolumn{5}{c}{ Tempo após a administração de $M$. rigida } \\
\cline { 2 - 6 } & T0 & T1 & T3 & T5 & T7 \\
\hline G1 & $0,315 \pm 0,02 \mathrm{aA}$ & $0,315 \pm 0,03 \mathrm{aA}$ & $0,297 \pm 0,03 \mathrm{aA}$ & $0,300 \pm 0,03 \mathrm{aAB}$ & $0,298 \pm 0,03 \mathrm{aA}$ \\
$\mathrm{G} 2$ & $0,303 \pm 0,02 \mathrm{aA}$ & $0,311 \pm 0,03 \mathrm{aA}$ & $0,293 \pm 0,05 \mathrm{aA}$ & $0,334 \pm 0,03 \mathrm{aA}$ & $0,340 \pm 0,02 \mathrm{aA}$ \\
$\mathrm{G} 3$ & $0,304 \pm 0,01 \mathrm{aA}$ & $0,259 \pm 0,01 \mathrm{bB}$ & $0,237 \pm 0,03 \mathrm{bA}$ & $0,271 \pm 0,025 \mathrm{abB}$ & $0,300 \pm 0,034 \mathrm{aA}$ \\
\hline
\end{tabular}

Médias seguidas por letras distintas, maiúsculas na coluna e minúsculas na linha, diferem entre si pelo teste Duncan, $(\mathrm{P}<0,05)$. CV: 7,8\%.

T0: dia zero; T1: um dia; T3: três dias; T5: cinco dias; T7: sete dias.

Não houve diferença estatística entre as médias dos intervalos RR dos grupos, registrados nos eletrocardiogramas, em repouso ou após exercício físico (Tab. 6 e 7).

Considerando todas as análises realizadas nos traçados eletrocardiográficos, pode-se concluir que $20 \mathrm{~g} / \mathrm{kg}$ de folhas de $M$. rigida, trituradas com água e administradas via oral, aumentaram significativamente a FC dos animais intoxicados, sobretudo durante o esforço físico, o que poderia predispor a arritmias cardíacas. Este resultado pode ser útil na explicação das mortes súbitas causadas por essa planta. O fato de não terem sido observadas alterações nos traçados pode ser porque elas ocorrem somente momentos antes da morte, sendo necessário monitoramento constante para detectá-las (Diniz et al., 2007).

Em relação aos exames ecodopplercardiográficos, imagens de boa qualidade foram obtidas com os animais em decúbito lateral (Cavalcanti et al., 2007), utilizando-se as regiões paraesternais direita e esquerda, do terceiro ao quinto espaços intercostais, sendo este posicionamento utilizado nas pesquisas com ovelhas (Dodic et al., 2001; Huang et al., 2004).

Tabela 6. Intervalo R-R (segundos) de ovinos do grupo-controle (G1) e de ovinos que receberam suspensão aquosa de $M$. rigida Griseb consecutivamente durante três dias (G2) e sete dias (G3), em repouso

\begin{tabular}{cccccc}
\hline \multirow{2}{*}{ Grupo } & \multicolumn{5}{c}{ Tempo após a administração de $M$. rigida } \\
\cline { 2 - 6 } & T0 & T1 & T3 & T5 & T7 \\
\hline G1 & $0,661 \pm 0,10 \mathrm{aA}$ & $0,644 \pm 0,12 \mathrm{aA}$ & $0,531 \pm 0,05 \mathrm{aA}$ & $0,694 \pm 0,19 \mathrm{aA}$ & $0,565 \pm 0,095 \mathrm{aA}$ \\
G2 & $0,578 \pm 0,03 \mathrm{aA}$ & $0,531 \pm 0,13 \mathrm{abA}$ & $0,406 \pm 0,10 \mathrm{bA}$ & $0,60 \pm 0,10 \mathrm{aA}$ & $0,657 \pm 0,13 \mathrm{aA}$ \\
G3 & $0,611 \pm 0,12 \mathrm{aA}$ & $0,472 \pm 0,10 \mathrm{aA}$ & $0,480 \pm 0,13 \mathrm{aA}$ & $0,492 \pm 0,083 \mathrm{aA}$ & $0,634 \pm 0,086 \mathrm{aA}$ \\
\hline
\end{tabular}

Médias seguidas por letras distintas, maiúsculas na coluna e minúsculas na linha, diferem entre si pelo teste Duncan, $(\mathrm{P}<0,05)$. CV: $14,5 \%$.

T0: dia zero; T1: um dia; T3: três dias; T5: cinco dias; T7: sete dias.

Tabela 7. Intervalo R-R (segundos) de ovinos do grupo-controle (G1) e de ovinos que receberam suspensão aquosa de $M$. rigida Griseb consecutivamente durante três dias (G2) e sete dias (G3), após 10 minutos de exercício

\begin{tabular}{cccccc}
\hline \multirow{2}{*}{ Grupo } & \multicolumn{5}{c}{ Tempo após a administração de $M$. rigida } \\
\cline { 2 - 6 } & T0 & T1 & T3 & T5 & T7 \\
\hline G1 & $0,488 \pm 0,07 \mathrm{aA}$ & $0,466 \pm 0,08 \mathrm{aA}$ & $0,447 \pm 0,06 \mathrm{aA}$ & $0,508 \pm 0,06 \mathrm{aA}$ & $0,220 \pm 0,02 \mathrm{bB}$ \\
G2 & $0,471 \pm 0,04 \mathrm{aA}$ & $0,363 \pm 0,03 \mathrm{bB}$ & $0,334 \pm 0,05 \mathrm{bB}$ & $0,420 \pm 0,11 \mathrm{aA}$ & $0,546 \pm 0,097 \mathrm{aA}$ \\
G3 & $0,516 \pm 0,12 \mathrm{aA}$ & $0,384 \pm 0,06 \mathrm{bcAB}$ & $0,331 \pm 0,04 \mathrm{cB}$ & $0,401 \pm 0,06 \mathrm{abcA}$ & $0,490 \pm 0,12 \mathrm{abA}$ \\
\hline
\end{tabular}

Médias seguidas por letras distintas, maiúsculas na coluna e minúsculas na linha, diferem entre si pelo teste Duncan, $(\mathrm{P}<0,05)$. CV: $14,2 \%$.

T0: dia zero; T1: um dia; T2: dois dias; T3: três dias; T4: quatro dias; T5: cinco dias; T6: seis dias; T7: sete dias. 
No modo bidimensional não foram observadas anormalidades nas valvas, nos grandes vasos e nas câmaras cardíacas em nenhum animal. Em experimentos com ovelhas, em que a função cardíaca foi significativamente afetada, também não foram relatadas anormalidades neste modo (Devlin et al., 2000; Moainie et al., 2002; Huang et al., 2004; Borenstein et al., 2006; Rabbani et al., 2006). Da mesma forma, no modo Doppler, não foram detectadas alterações que indicassem obstrução, regurgitação ou turbulência do fluxo sanguíneo.
Não foram observadas diferenças significativas entre os grupos no que diz respeito às mensurações dimensionais obtidas no modo-M (Tab. 8 a 14), permitindo inferir que $M$. rigida não causou dilatação ou hipertrofia, visto que as dimensões mantiveram-se dentro da faixa de normalidade para a espécie (Moses e Ross, 1987). Em modelos de insuficiência cardíaca crônica em ovelhas, alterações significativas nestas dimensões só foram observadas após 30 dias (Devlin et al., 2000; Huang et al., 2004).

Tabela 8. Espessura ( $\mathrm{mm}$ ) do septo em sístole de ovinos do grupo-controle (G1) e de ovinos que receberam suspensão aquosa de $M$. rigida Griseb consecutivamente durante três dias (G2) e sete dias (G3)

\begin{tabular}{cccc}
\hline \multirow{2}{*}{ Grupo } & \multicolumn{3}{c}{ Tempo após a administração de $M$. rigida } \\
\cline { 2 - 4 } & T0 & T3 & T7 \\
\hline G1 & $10,77 \pm 0,87$ & $10,59 \pm 1,38$ & $12,06 \pm 1,40$ \\
G2 & $9,37 \pm 0,73$ & $10,34 \pm 1,77$ & $10,89 \pm 1,24$ \\
G3 & $9,34 \pm 1,40$ & $9,71 \pm 0,97$ & $9,78 \pm 0,69$ \\
\hline
\end{tabular}

Não houve diferença estatística (P>0,05) pelo teste Duncan. CV: 12,2\%.

T0: dia zero; T3: três dias; T7: sete dias.

Tabela 9. Espessura (mm) do septo em diástole de ovinos do grupo-controle (G1) e de ovinos que receberam suspensão aquosa de $M$. rigida Griseb consecutivamente durante três dias (G2) e sete dias (G3)

\begin{tabular}{cccc}
\hline \multirow{2}{*}{ Grupo } & \multicolumn{3}{c}{ Tempo após a administração de $M$. rigida } \\
\cline { 2 - 4 } & T0 & T3 & T7 \\
\hline G1 & $7,17 \pm 1,00$ & $7,23 \pm 0,69$ & $7,50 \pm 0,75$ \\
G2 & $6,85 \pm 0,52$ & $8,13 \pm 0,97$ & $7,52 \pm 0,80$ \\
G3 & $7,63 \pm 0,78$ & $7,67 \pm 1,08$ & $7,75 \pm 1,03$ \\
\hline
\end{tabular}

Não houve diferença estatística (P>0,05) pelo teste Duncan. CV: 10,3\%.

T0: dia zero; T3: três dias; T7: sete dias.

Tabela 10. Diâmetro (mm) interno do ventrículo esquerdo em sístole de ovinos do grupo-controle (G1) e de ovinos que receberam suspensão aquosa de $M$. rigida Griseb consecutivamente durante três dias (G2) e sete dias (G3)

\begin{tabular}{cccc}
\hline \multirow{2}{*}{ Grupo } & \multicolumn{3}{c}{ Tempo após a administração de M. rigida } \\
\cline { 2 - 4 } & T0 & T3 & T7 \\
\hline G1 & $20,50 \pm 2,23$ & $21,16 \pm 2,15$ & $20,98 \pm 1,65$ \\
G2 & $21,18 \pm 1,58$ & $23,22 \pm 4,16$ & $19,20 \pm 1,91$ \\
G3 & $21,66 \pm 2,72$ & $22,34 \pm 3,80$ & $23,36 \pm 2,06$ \\
\hline
\end{tabular}

Não houve diferença estatística (P>0,05) pelo teste Duncan. CV: 10,6\%.

T0: dia zero; T3: três dias; T7: sete dias.

Tabela 11. Diâmetro (mm) interno do ventrículo esquerdo em diástole de ovinos do grupo-controle (G1) e de ovinos que receberam suspensão aquosa de $M$. rigida Griseb consecutivamente durante três dias $(\mathrm{G} 2)$ e sete dias (G3)

\begin{tabular}{cccc}
\hline \multirow{2}{*}{ Grupo } & \multicolumn{3}{c}{ Tempo após a administração de M. rigida } \\
\cline { 2 - 4 } & T0 & T3 & T7 \\
\hline G1 & $33,62 \pm 2,08$ & $33,92 \pm 2,6$ & $35,80 \pm 2,46$ \\
G2 & $33,8 \pm 1,44$ & $34,54 \pm 2,65$ & $33,12 \pm 1,17$ \\
G3 & $33,50 \pm 2,63$ & $33,40 \pm 1,82$ & $33,46 \pm 1,90$ \\
\hline
\end{tabular}

Não houve diferença estatística $(\mathrm{P}>0,05)$ pelo teste Duncan. CV: 5,0\%.

T0: dia zero; T3: três dias; T7: sete dias. 
Tabela 12. Espessura (mm) da parede do ventrículo esquerdo em sístole de ovinos do grupo-controle (G1) e de ovinos que receberam suspensão aquosa de $M$. rigida Griseb consecutivamente durante três dias (G2) e sete dias (G3)

\begin{tabular}{cccc}
\hline \multirow{2}{*}{ Grupo } & \multicolumn{3}{c}{ Tempo após a administração de $M$. rigida } \\
\cline { 2 - 4 } & T0 & T3 & T7 \\
\hline G1 & $11,35 \pm 0,88$ & $11,20 \pm 1,00$ & $12,28 \pm 1,51$ \\
G2 & $11,08 \pm 0,33$ & $11,56 \pm 1,20$ & $11,37 \pm 1,50$ \\
G3 & $10,84 \pm 1,09$ & $10,68 \pm 1,00$ & $10,19 \pm 1,25$ \\
\hline
\end{tabular}

Não houve diferença estatística (P>0,05) pelo teste Duncan. CV: 10,2\%.

T0: dia zero; T3: três dias; T7: sete dias.

Tabela 13. Espessura $(\mathrm{mm})$ da parede do ventrículo esquerdo em diástole de ovinos do grupo-controle (G1) e de ovinos que receberam suspensão aquosa de $M$. rigida Griseb consecutivamente durante três dias (G2) e sete dias (G3)

\begin{tabular}{cccc}
\hline \multirow{2}{*}{ Grupo } & \multicolumn{3}{c}{ Tempo após a administração de $M$. rigida } \\
\cline { 2 - 4 } & T0 & T3 & T7 \\
\hline G1 & $6,03 \pm 1,05$ & $6,89 \pm 0,81$ & $5,42 \pm 0,60$ \\
G2 & $6,92 \pm 0,71$ & $7,94 \pm 1,46$ & $5,79 \pm 1,13$ \\
G3 & $6,77 \pm 1,01$ & $6,88 \pm 0,60$ & $6,44 \pm 0,72$ \\
\hline
\end{tabular}

Não houve diferença estatística $(\mathrm{P}>0,05)$ pelo teste Duncan. CV: $11.2 \%$.

T0: dia zero; T3: três dias; T7: sete dias.

Tabela 14. Separação septal do ponto E (mm) da válvula mitral de ovinos do grupo-controle (G1) e de ovinos que receberam suspensão aquosa de $M$. rigida Griseb consecutivamente durante três dias $(\mathrm{G} 2)$ e sete dias (G3)

\begin{tabular}{cccc}
\hline \multirow{2}{*}{ Grupo } & \multicolumn{3}{c}{ Tempo após a administração de $M$. rigida } \\
\cline { 2 - 4 } & T0 & T3 & T7 \\
\hline G1 & $4,89 \pm 0,62$ & $3,71 \pm 1,16$ & $5,08 \pm 0,74$ \\
G2 & $4,13 \pm 0,37$ & $4,50 \pm 0,24$ & $4,34 \pm 0,27$ \\
G3 & $3,69 \pm 0,34$ & $4,48 \pm 0,51$ & $4,49 \pm 0,44$ \\
\hline
\end{tabular}

Não houve diferença estatística $(\mathrm{P}>0,05)$ pelo teste Duncan. $\mathrm{CV}: 15,8 \%$

T0: dia zero; T3: três dias; T7: sete dias.

Para os índices funcionais estimados pelo ModoM (Tab. 15 e 16), não houve diferença entre os grupos no que se refere aos percentuais de encurtamento sistólico do ventrículo esquerdo (Tab. 15) ou às frações de ejeção (Tab. 16). Duas observações devem ser consideradas: a primeira é que as médias sugerem uma redução nos índices nos animais tratados, sobretudo nos animais que receberam a planta durante sete dias (G3); a segunda é que elas também sugerem uma recuperação nos índices após a última administração da planta, como observado nos animais tratados por três dias (G2).

Para o percentual de encurtamento sistólico (Tab. 15), a comparação com a literatura reforça o que foi sugerido acima, pois ele foi mais baixo em
G2 e G3 em relação aos valores normais de 37,2\% (Moses e Ross, 1987), 42\% (Dodic et al., 2001), 40\% (Rabbani et al., 2006). No presente experimento, percentual de encurtamento sistólico foi apenas $32,4 \%$ no G2 e menor ainda, $29,5 \%$, no G3 ao fim da administração da planta. As médias do G1 mantiveram-se semelhantes aos valores normais.

A fração de ejeção (Tab. 16) também foi menor nos animais tratados em relação aos valores normais de literatura, apenas $63 \%$ no G2 e $54,6 \%$, no G3 ao fim da administração da planta. As médias do G1 foram semelhantes aos valores de $68 \%$ e $71 \%$ obtidos de ovelhas normais (Devlin et al., 2000; Rabbani et al., 2006). 
Tabela 15. Percentual de encurtamento sistólico do ventículo esquerdo (\%) de ovinos do grupo-controle (G1) e de ovinos que receberam suspensão aquosa de M. rigida Griseb consecutivamente durante três dias (G2) e sete dias (G3)

\begin{tabular}{cccc}
\hline \multirow{2}{*}{ Grupo } & \multicolumn{3}{c}{ Tempo após a administração de $M$. rigida } \\
\cline { 2 - 4 } & T0 & T3 & T7 \\
\hline G1 & $39,04 \pm 4,42$ & $37,61 \pm 5,19$ & $41,34 \pm 1,40$ \\
G2 & $37,28 \pm 4,50$ & $32,42 \pm 6,25$ & $41,97 \pm 5,80$ \\
G3 & $35,48 \pm 3,91$ & $33,30 \pm 9,63$ & $29,52 \pm 6,50$ \\
\hline
\end{tabular}

Não houve diferença estatística (P>0,05) pelo teste Duncan. CV: $12,5 \%$.

T0: dia zero; T3: três dias; T7: sete dias.

Tabela 16. Fração de ejeção do ventrículo esquerdo (\%) de ovinos do grupo-controle (G1) e de ovinos que receberam suspensão aquosa de $M$. rigida Griseb consecutivamente durante três dias (G2) e sete dias (G3)

\begin{tabular}{cccc}
\hline \multirow{2}{*}{ Grupo } & \multicolumn{3}{c}{ Tempo após a administração de $M$. rigida } \\
\cline { 2 - 4 } & T0 & T3 & T7 \\
\hline G1 & $70,44 \pm 5,32$ & $68,60 \pm 6,37$ & $73,14 \pm 1,75$ \\
G2 & $68,10 \pm 5,64$ & $63,38 \pm 9,87$ & $73,07 \pm 6,13$ \\
G3 & $66,38 \pm 5,54$ & $60,72 \pm 15,69$ & $54,64 \pm 10,47$ \\
\hline
\end{tabular}

Não houve diferença estatística $(\mathrm{P}>0,05)$ pelo teste Duncan. CV: $9.4 \%$

T0: dia zero; T3: três dias; T7: sete dias.

Redução na fração de encurtamento e a na fração de ejeção ocorrem nos estágios iniciais da insuficiência cardíaca e indicam que a função ventricular está sendo afetada (Kittleson e Kienle, 1998; Silva et al., 2008). A despeito da possibilidade de ter havido comprometimento cardíaco pela administração de $M$. rígida, a taxa de mortalidade foi baixa nos animais tratados. Uma explicação para isso pode ser a de que, quando a função ventricular diminui, uma hierarquia de sistemas neuro-humorais é estimulada para manter a perfusão tecidual nos órgãos vitais (Charles et al., 2003).

Os resultados ecodopplercardiográficos indicam que $M$. rigida acomete a função cardíaca, demonstrado pela redução na fração de ejeção e na fração de encurtamento sistólico dos ovinos durante a administração da planta, nas condições deste experimento. Somando-se a isso o aumento causado na FC, pode-se pressupor que, em animais mais gravemente intoxicados, um possível mecanismo para a morte súbita seja a baixa oxigenação do miocárdio devido à redução relativa no fluxo coronário, associada ao maior consumo de oxigênio em situações de grande esforço físico.

\section{REFERÊNCIAS BIBLIOGRÁFICAS}

BOON, J.A. Manual of veterinary echocardiography. Baltimore: Williams \& Wilkins, 1998. 261p.

BORENSTEIN, N.; BRUNEVAL, P.; BEHR, L. et al. An ovine model of chronic heart failure: echocardiographic and tissue Doppler imaging characterization. J. Card. Surg., v.21, p.50-56, 2006.

BOTHA, C.J.; VAN DER LUGT, J.J.; ERASMUS G.L. et al. Krimpsiekte associated with thalamic lesions, induced by the neurotoxic cardiac glycoside, cotyledoside, isolated from Tylecodon wallichii (Harv.) toelken subsp. wallichii. Onderstepoort J. Vet. Res., v.64, p.189-194, 1997.

CARVALHO, P.S.L.; PEREIRA, G.G; PETRUS, L.C. et al. Avaliação de alguns parâmetros ecocardiográficos do gato-do-mato (Leopardus tigrinus) mantido em cativeiro e submetido à anestesia com xilazina e quetamina. Arq. Bras. Med. Vet. Zootec., v.59, p.695-699, 2007.

CARVALHO, R.O.; ARAÚJO, R.B.; SILVA, E.F. Ecocardiografia modo Doppler pulsado em 
gatos clinicamente sadios. Arq. Bras. Med. Vet. Zootec., v.58, p.333-340, 2006.

CAVALCANTI, G.A.O.; MUZZI, R.A.L.; ARAÚJO, R.B. et al. Avaliação ecodopplercardiográfica da função diastólica em cães da raça Boxer. Arq. Bras. Med. Vet. Zootec., v.59, p.1169-1176, 2007.

CHARLES, J.C.; ELLIOTT, J.M.; NICHOLLS, M.G. et al. Natriuretic peptides maintain sodium homoeostasis during chronic volume loading post-myocardial infarction in sheep. Clin. Sci., v.104, p.429-436, 2003.

DEVLIN, G.; MATTHEWS, K.; McCRACKEN, G. et al. An ovine model of chronic stable heart failure. J. Card. Fail, v.6, p.140-143, 2000.

DINIZ, M.P.; MUZZI, R.A.L.; MUZZI, L.A.L. et al. Estudo eletrocardiográfico de eqüinos da raça Mangalarga Marchador. Arq. Bras. Med. Vet. Zootec., v.60, p.536-542, 2008.

DINIZ, P.P.V.P.; SCHWARTZ, D.S.; COLLICCHIO-ZUANAZE, R.C. Cardiac trauma confirmed by cardiac markers in dogs: two case reports. Arq. Bras. Med. Vet. Zootec., v.59, p.8589, 2007.

DODIC, M.; SAMUEL, C.; MORITZ, K. et al. Impaired cardiac functional reserve and left ventricular hypertrophy in adult sheep after prenatal dexamethasone exposure. Circ. Res., v.89, p.623-629, 2001.

FRIDERICIA, L.S. Die systolendauer im elektrokardiogramm bei normalen menschen und bei herzkranken. Acta Med. Scand., v.53, p.469486, 1920.

GAVA, A.; CRISTANI, J.; BRANCO, J.V. et al. Mortes súbitas em bovinos causadas pela ingestão de Mascagnia sp. (Malpighiaceae) no Estado de Santa Catarina. Pesq. Vet. Bras., v.18, p.16-20, 1998.

HUANG, Y.; HUNYOR, S.N.; JIANG, L. et al. Remodeling of the chronic severely failing ischemic sheep heart after coronary microembolization: functional, energetic, structural and cellular responses. Am. J. Physiol. Heart. Circ. Physiol., v.286, p.2141-2150, 2004.

KER, J.; WEBB, E.C.; KER, J.A. et al. The heart remembers: observations of cardiac memory in the Doper sheep heart. J. Vet. Res., v.70, p.299305, 2003.
KITTLESON, M.D.; KIENLE, R.D. Small animal cardiovascular medicine. St. Louis: Mosby, 1998. 603p.

KOYAMA, H.; YOSHII, H.; YABU, H. et al. Evaluation of QT interval prolongation in dogs with heart failure. J. Vet. Med. Sci., v.66, p.11071111, 2004.

MELO, M.M.; VERÇOSA JÚNIOR, D.; PINTO, M.C.L. et al. Intoxicação experimental com extratos de Mascagnia rigida. Arq. Bras. Med. Vet. Zootec., v.60, p.631-640, 2008.

MIR, S.A.; NAZKI, A.R.; RAINA, R. Comparative electrocardiographic studies, and differing effects of pentazocine on ECG, heart and respiratory rates in young sheep and goats. Small Rumin. Res., v.37, p.13-17, 2000.

MOAINIE, S.L.; GORMAN III, J.H.; GUY, T.S. et al. An ovine model of postinfarction dilated cardiomyopathy. Ann. Thorac. Surg., v.74, p.753-760, 2002.

MOSES, B.L.; ROSS Jr., J.N. M-mode echocardiographic values in sheep. Am. J. Vet. Res., v.48, p.1313-1318, 1987.

PARAGUASSÚ, A.A. Intoxicação experimental por Mascagnia rígida (Malpighiaceae) em caprinos no nordeste do Brasil. 1983. 65f. Dissertação (Mestrado) - Universidade Federal Rural do Rio de Janeiro, Itaguaí, RJ.

PINHEIRO, D.C.S.N.; PEREIRA, A.S.; SANTOS, L.F.L. Intoxicação experimental em caprinos sem raça definida com tingüi (Mascagnia rigida Malpighiaceae). In: CONGRESSO BRASILEIRO DE MEDICINA VETERINÁRIA, 1996, Goiânia. Anais... Goiânia: 1996. v.1, p.14.

PUGH, D.G. Sheep \& goat medicine. Philadelphia: Saunders, 2002. 468p.

RABBANI, S.; AHMADI, H.; FAYAZZADEH, E. et al. Induced myocardial infarction using ligation of the left anterior descending coronary artery major diagonal branch: development of an ovine model. J. Tehran Heart Center, v.1, p.8993, 2006.

SCHULTZ, R.A.; COETZER, J.A.W.; KELLERMAN, T.S. et al. Observations on the clinical, cardiac and histopathological effects of fluoroacetate in sheep. Onderstepoort J. Vet. Res., v.49, p.237-245, 1982. 
SCHULTZ, R.A.; PRETORIUS, P.J.; TERBLANCHE, M. An electrocardiographic study of normal sheep using a modified technique. Onderstepoort J. Vet. Res., v.39, p.97106, 1972.

SILVA, E.F.; MELO, M.B.; MUZZI, R.A.L. et al. Índices ecodopplercardiográficos da função ventricular esquerda em cães da raça Boxer e Schnauzer Miniatura. Arq. Bras. Med. Vet. Zootec., v.60, p.71-75, 2008.

TILLEY, L.P. Essentials of canine and feline electrocardiography: interpretation and treatment. 3.ed. Philadelphia: Lea \& Febiger, 1992. 470p.
TOKARNIA, C.H.; DÖBEREINER, J.; PEIXOTO, P.V. Aspectos clínico-patológicos complementares da intoxicação por algumas plantas tóxicas brasileiras. Pesq. Vet. Bras., v.14, p.111-122, 1994.

TOKARNIA, C.H.; DÖBEREINER, J.; PEIXOTO, P.V. Plantas tóxicas do Brasil. Rio de Janeiro: Helianthus, 2000. 220p.

TÓRIO, R.; CANO, M.; MONTES, A. et al. Comparison of two methods for electrocardiographic analysis in Gallega sheep. Small Rumin. Res., v.24, p.239-246, 1997. 